\title{
Flicker control in mains with distributed generation plants
}

\author{
Yuri Bulatov ${ }^{1, *}$, Andrey Kryukov ${ }^{2,3}$, and Nguyen Van Huan ${ }^{3}$ \\ ${ }^{1}$ Bratsk State University, Bratsk, Russia \\ ${ }^{2}$ Irkutsk State Transport University, Irkutsk, Russia \\ ${ }^{3}$ Irkutsk National Research Technical University, Irkutsk, Russia
}

\begin{abstract}
The use of distributed generation (DG) plants in power supply systems is a rapid development line. However, the impact of DG on power quality is multivalued. On the one hand, the presence of DG allows to reduce voltage losses. On the other hand, a phenomenon called flicker and associated with rapid voltage fluctuations is possible. This effect is usually manifested at an abrupt voltage drop in the DG generator connection unit. The processes taking place in the network when flicker occurs in networks with DG have not been sufficiently studied. The article presents results of the flicker modeling in a network equipped with DG plants, implemented on the basis of synchronous generators. The results obtained indicated that with sharp disturbances caused by switching on and off an additional load, flicker is observed in networks with unregulated generators, accompanied by voltage and frequency fluctuations. Based on the wavelet transformation and spectral analysis methods, it was found that the power spectral density of the generated flicker-noise is inversely proportional to the frequency. The use of look-ahead control algorithms to control the excitation and rotors rotational speed of the DG plants generators, as well as concordant adjustment of their controllers, increases stability and removes flicker completely.
\end{abstract}

\section{Introduction}

Distributed generation (DG) plants are broadly used nowadays to develop and upgrade power supply systems (PSS), but there are power sources which are located in close proximity from the consumers and whose operation is based on the different technologies: wind power generators, solar batteries, fuel cells, gas turbine power plants, mini- and micro-HPPs, etc.

Distributed power generation is a counterpart of Smart Grids concept [1-3] and can be used for mains load shedding, power and energy losses reduction and enhancing PSS reliability and survivability. New electrical power markets can be generated using DG plants [4]. It should be noted the distributed generation can produce unambiguous effect on PSS power quality. DG plants allow to maintain the required voltage levels in the mains nodes $[5,6]$, reduce unsymmetry and harmonic distortions in PSS [7]. However, low-power generators can cause voltage fluctuations which cause flicker in some cases [4, 8-10]. This can occur in case of an abrupt voltage drop in the connection node of DG plant. Incorrectly adjusted automatic voltage regulators (AVR) and automatic speed regulators (ASR) of DG plants can enhance the occurring flicker [10]. The processes taking place in DG plants mains on the background of arising flicker, have not been sufficiently studied. A broader study of the DG plants based on AVR and ASR generators require a precise assessment of their affect on PSS to eliminate power quality deterioration. The problems of flicker assessment and elimination in
PSS using the controlled DG plants are undoubtedly of urgent character.

The work provides the study results of PSS working modes with DG plants implemented based on synchronous generators with AVR and ASR. To analyze flicker-noise in the network under study, spectral analysis and wavelet transformation methods were used.

\section{Description of the network under study and synchronous generator regulators used}

The study was carried out in MATLAB system based on PSS models with DG plants. Diagram of the PSS under study is represented in fig. 1. A PSS was simulated with a total consumer load of $5+j 2.4 \mathrm{MV} \cdot \mathrm{A}$ connected to supplying electrical energy system (EES) (110 kV System unit in fig. 1) through a $110 / 35 / 6 \mathrm{kV}$ transformer. The PSS consisted of DG plants, implemented on the basis of two turbine generators (Synchronous Machine units) with a rated power of $3.125 \mathrm{MV} \cdot \mathrm{A}$ each and 6.3 $\mathrm{kV}$ voltage. The generators were simulated by the Synchronous Machine pu Fundamental units included in the SymPowerSystems library. Fig. 2 provides a structural diagram of the used steam turbine model (Steam turbine unit in fig. 1). The following turbogenerator parameters were used for modeling: the reactance of the machine along the longitudinal axis $X_{d}=2.34$ r.u.; generator EMF $E_{q}=1.25$ r.u; constant of the generator mechanical inertia $T j=8.669 \mathrm{~s}$, etc.

* Corresponding author: bulatovyura@yandex.ru 


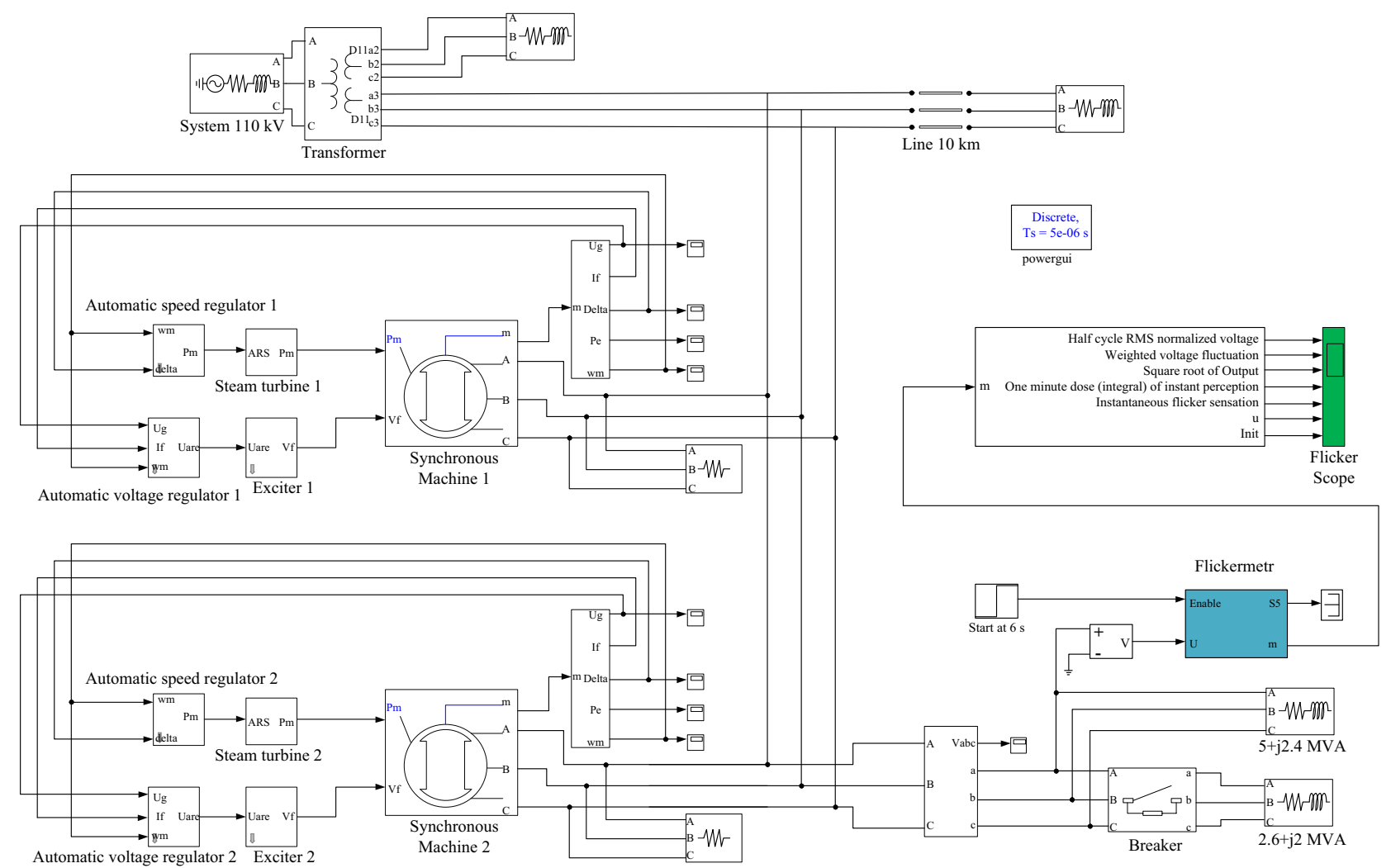

Fig. 1. The study model diagram in MATLAB.

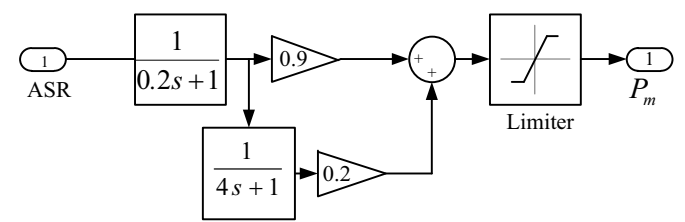

Fig. 2. Structural diagram of the used steam turbine model.

Models of thyristor excitation systems (Exciter1 and Exciter2 units) are implemented based on the equations provided in [11]. To control the rotor rotational frequency and voltage of the DG plants generators, the AVR and ASR models were used, which implement proportional-integral-differential (PID) control laws and are represented by the following transfer functions:

1) Automatic speed regulator unit:

$$
\left(k_{p}+\frac{k_{i}}{0.1 s}+\frac{k_{d} s}{s+1}\right) \cdot \frac{1}{0.01 s+1},
$$

where $k_{p}, k_{i}, k_{d}-\mathrm{ASR}$ adjustment coefficients; $\mathrm{s}-$ Laplasian operator;

2) Automatic voltage regulatoe unit:

$$
\frac{1+0.5 s}{0.5 s} \cdot\left(k_{0 u}-\frac{0.02 k_{1 u} s}{0.06 s+1}+\frac{2 k_{0 \omega} s}{(2 s+1)(0.02 s+1)}+\frac{0.05 k_{1 \omega} s}{0.05 s+1}\right)
$$

where $k_{0 u}, k_{1 u}, k_{0 \omega}$ and $k_{1 \omega}-$ coefficients of AVR channels adjustment.

The model also used look-ahead control algorithms a detailed description of which is provided in $[12,13]$. The structural diagram of auto prognostic ASR is provided in fig. 3 [13]. It included the following units:
- PID controller whose mathematical description is provided above;

- two links connected in parallel with a transfer function $\frac{K_{a} s}{T_{a} s+1}\left(K_{a}=1 ; T_{a}=0.001 \mathrm{~s}\right)$;

- unit for calculating $T p$ time constant of the linear forecasting link, which depends on the angle between the voltage and the generator EMF $\delta$.

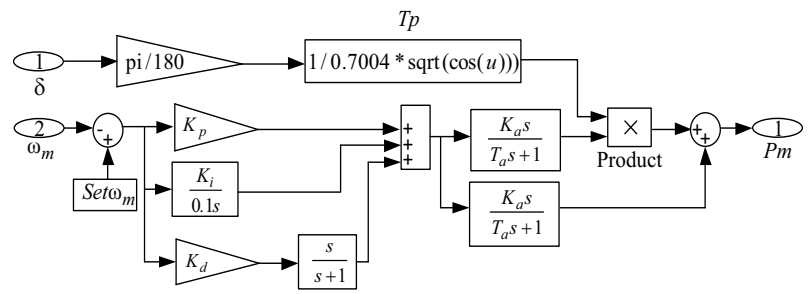

Fig. 3. Structural diagram of auto prognostic ASR.

Measurements of voltage and frequency fluctuations were performed using oscilloscopes. The modes leading to the occurrence of flicker were created by short-term connection to the node with additional load DG plants with a power of $2.6+j 2 \mathrm{MV} \cdot \mathrm{A}$.

The model used a standard Flickermetr unit (fig. 1), which implements a digital flickermeter in accordance with the international standard IEC 61000-4-15. It allowed measuring the following parameters: root-meansquare voltage for each half cycle; weighted voltage fluctuation obtained after passing through a special filter; integral one-minute flicker dose; instant flicker sensation (instant flicker dose). 


\section{Description of the study results}

The studies were carried out for the following operating modes of DG plants generators: without regulators; with concordantly and non-concordantly configured AVR and ASR; using predictive algorithms in AVR and ASR.

The computational experiments carried out on the model indicated that in case of operation of small turbine generators without AVR and ASR, there is a high probability of stability loss and the occurrence of an asynchronous run when an additional load is connected, which causes a decrease in voltage in the DG plants connection assembly. In this case, fluctuations of the generator rotor speed (fig. $4 \mathrm{a}$ ) and the voltage on the $6 \mathrm{kV}$ buses (fig. 4b) occur. These fluctuations can propagate across the entire network. It should be noted that Flickermetr shows the presence of flicker (fig. 5).

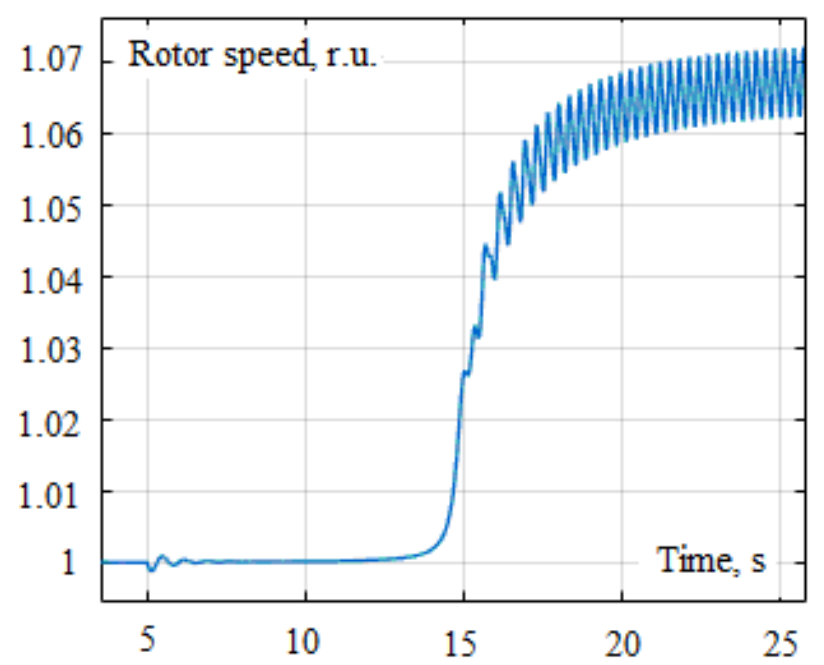

a)

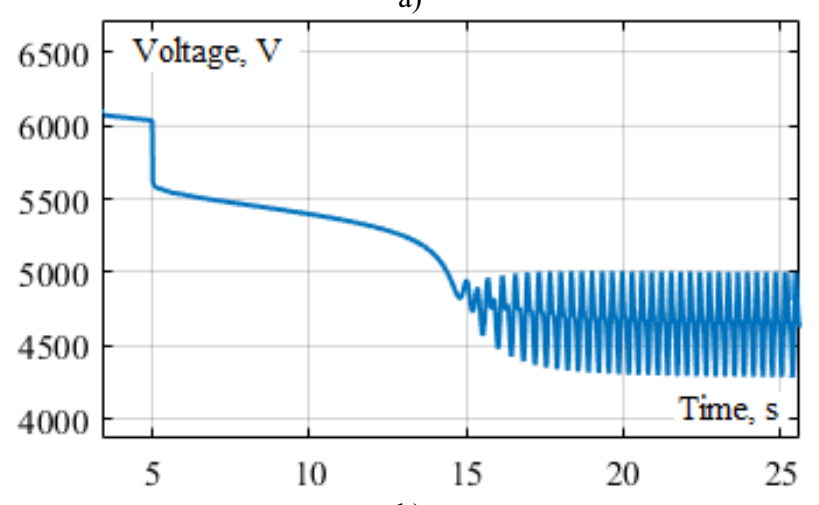

b)

Fig. 4. Oscillograms of rotor speed (a) and voltage (b) of generator without regulators when additional load is connected in the node.

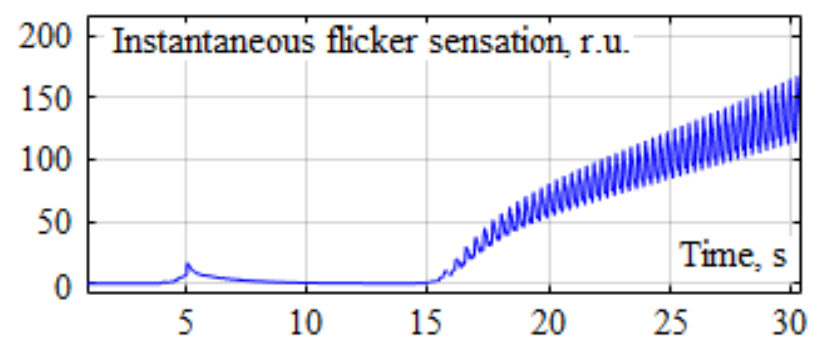

Fig. 5. Flickermeter reading (instantaneous flicker dose).
The resulting noise was selected from the signal of the effective voltage in the assembly with DG plants using the wavelet transformation. The user-selected noise is shown in fig. 6 .

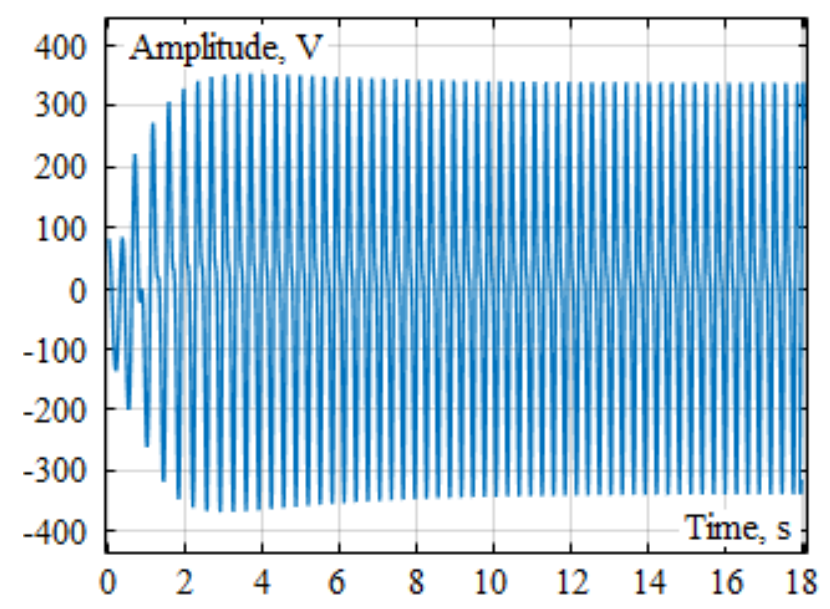

Fig. 6. User-selected noise.

The analysis results proved that the power spectral density of the selected noise is inversely proportional to the frequency. Dependences of spectral power density Spd on frequency at various scales, obtained using the Berg method [14], are provided in fig. 7, 8. Processing the results obtained indicates that

Spd $\sim \frac{1}{f^{\beta}}$,

where $\beta=3.59$ the spectrum shape index. Thus, the user-selected noise can be attributed to flicker-noise [15, $16]$.

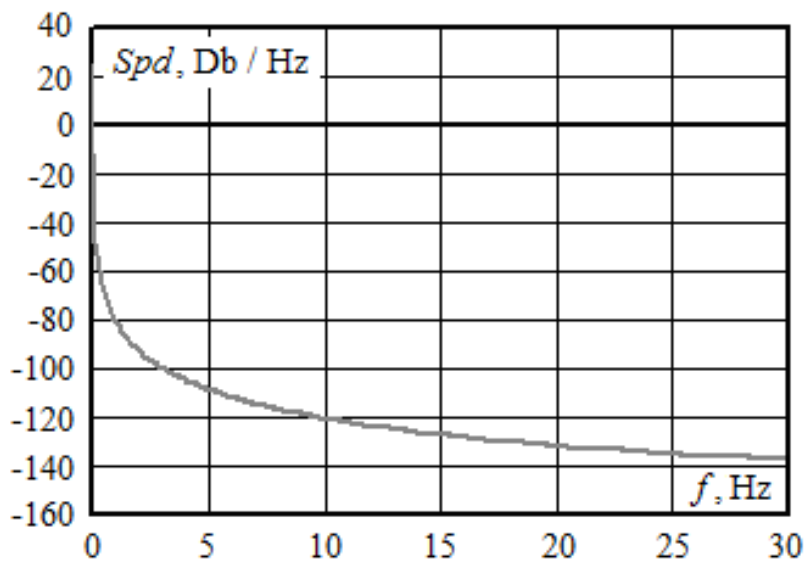

Fig. 7. Dependence of $\operatorname{Spd}(\mathrm{Db} / \mathrm{Hz})$ on frequency.

The reason for the flicker-noise appearance in the system under study can be attributed to low-frequency fluctuations in the rotational speed of the rotors of DG plants generators when a sharp disturbance occurs in the assembly of their connection. Similar effects are observed with non-optimal adjustment of the DG plants regulators. Fig. 9 shows the corresponding oscillograms of the rotor speed and the generator voltage with nonconcordantly adjusted AVR and ASR. 


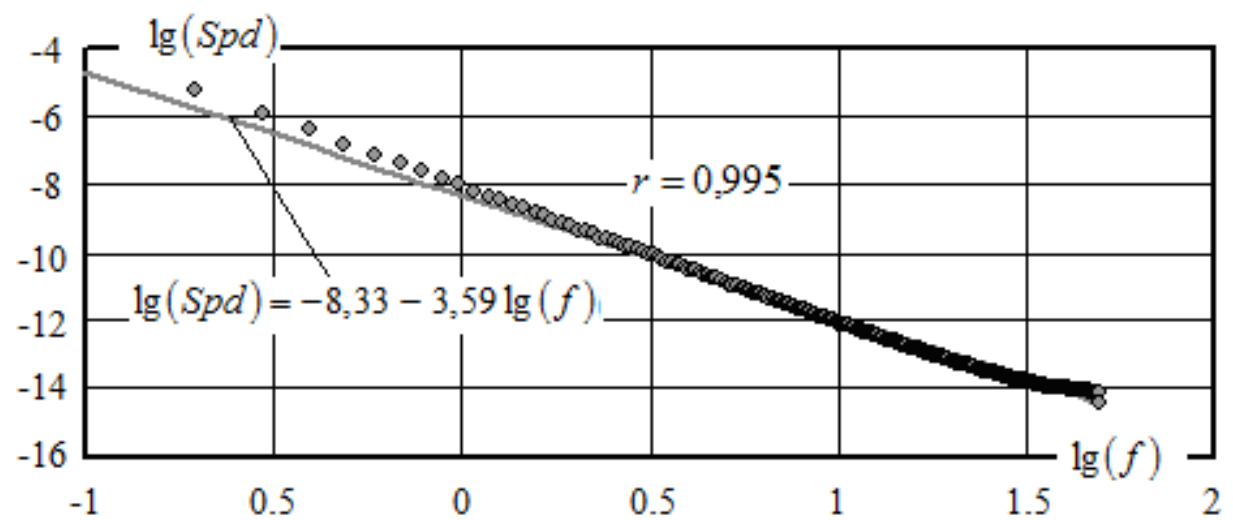

Fig. 8. Dependence of $\log (S p d)$ on frequency logarithm: $r$ - correlation coefficient.
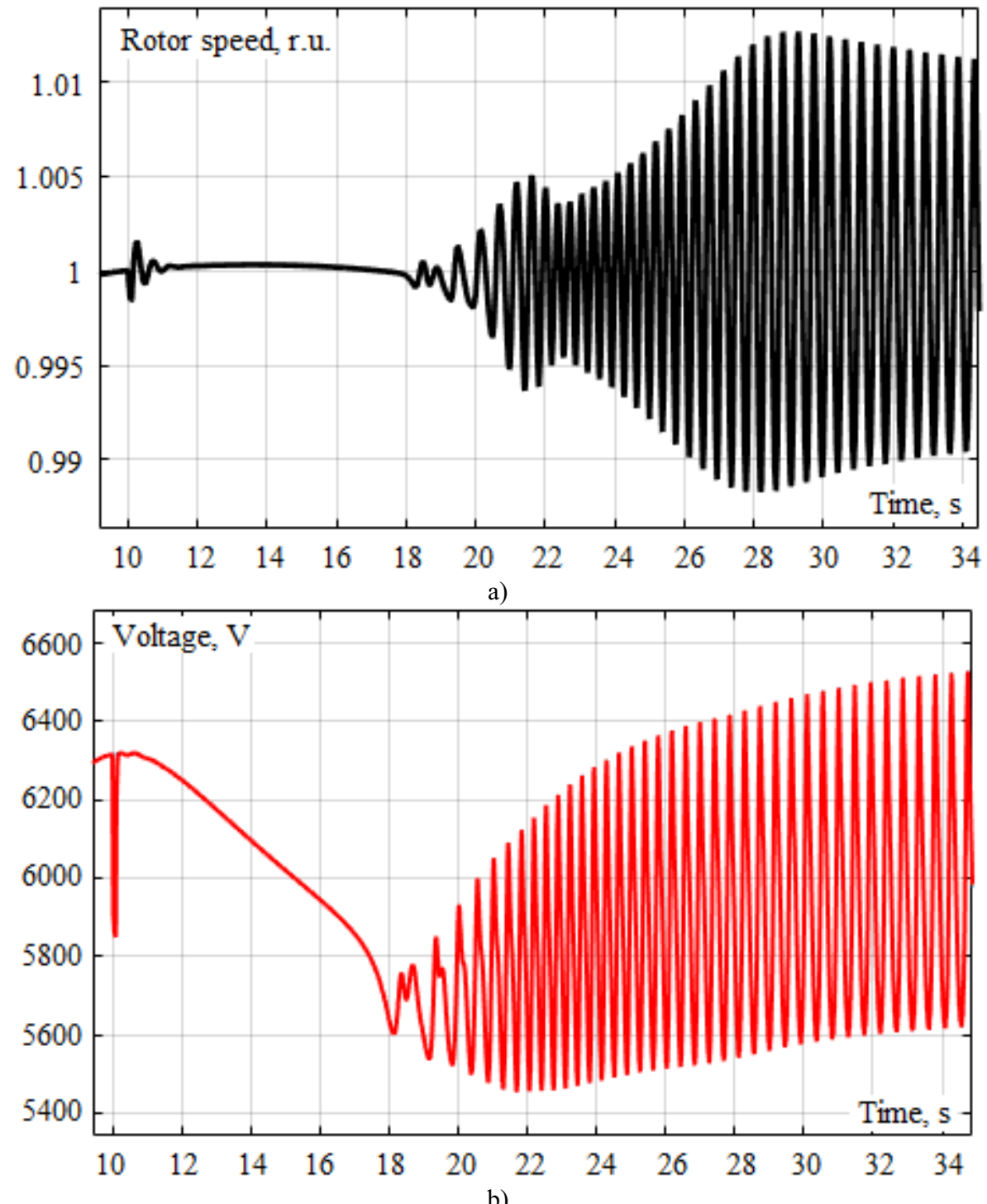

Fig. 9. Oscillations of the rotor speed (a) and voltage in the DG plant connection assembly (b) when a powerful load is switched on and off after $0.1 \mathrm{~s}$. Non-concordantly adjusted AVR and ASR were used.

It should be noted that the issues of flicker removal can be solved by correctly adjusting the DG plants regulators using standard techniques. More complex cases may require system measurements to evaluate voltage fluctuations and determine how controls can be adjusted or modified to reduce flicker-noise.

The studies conducted have proved that the use of look-ahead control algorithms to control the rotor speed and excitation of the DG plants turbine generators allows to increase stability and completely removes the occurrence of flicker even without using the regulators adjustment procedure. The corresponding oscillograms of the voltage and rotor speed of one of the generators with a sharp change in the consumer load are shown in fig. 10 and 11. The use of procedure for AVR and ASR concordant adjustment of synchronous generators [5], 
and look-ahead control algorithms helps to solve flicker occurrence issue. In this case, the quality indicators of transient processes in PSS equipped with DG plants are significantly improved. Oscillograms of the voltage and the generator rotor speed in case of a sharp change in the consumer load, as well as the readings of the flickermeter, confirming these conclusions, are shown in fig. 12 and 13.

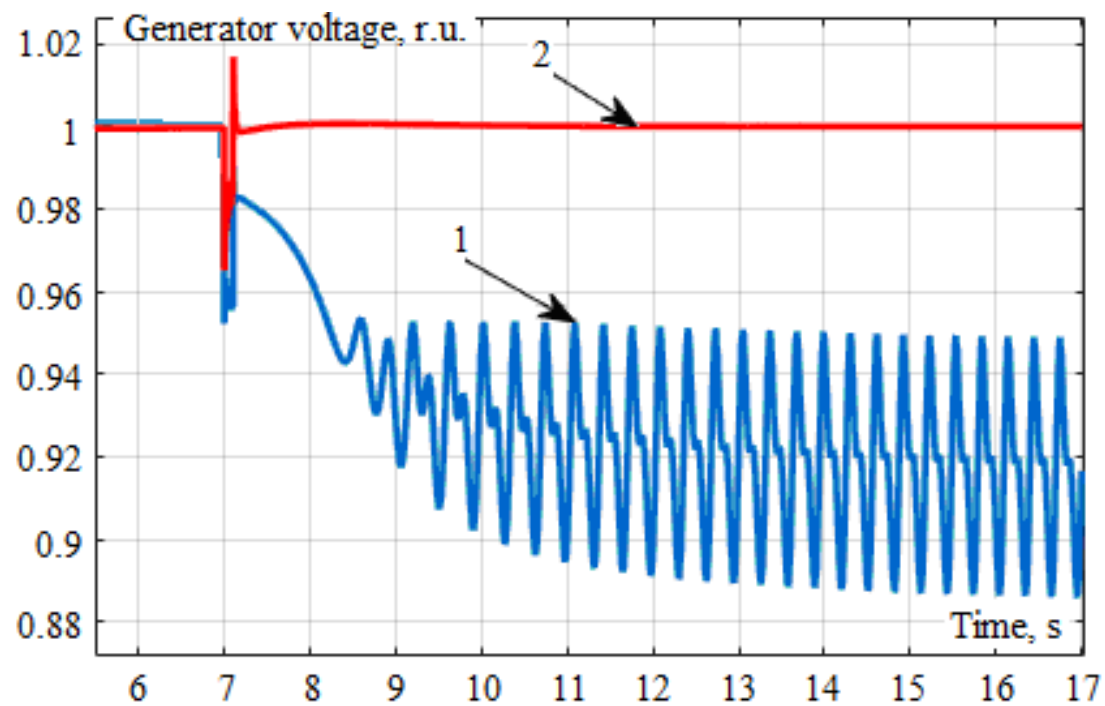

Fig. 10. Turbogenerator voltage oscillograms: 1 - generators were operated without AVR and ASR; 2 - generators were operated with the use of prognostic AVR and ASR.

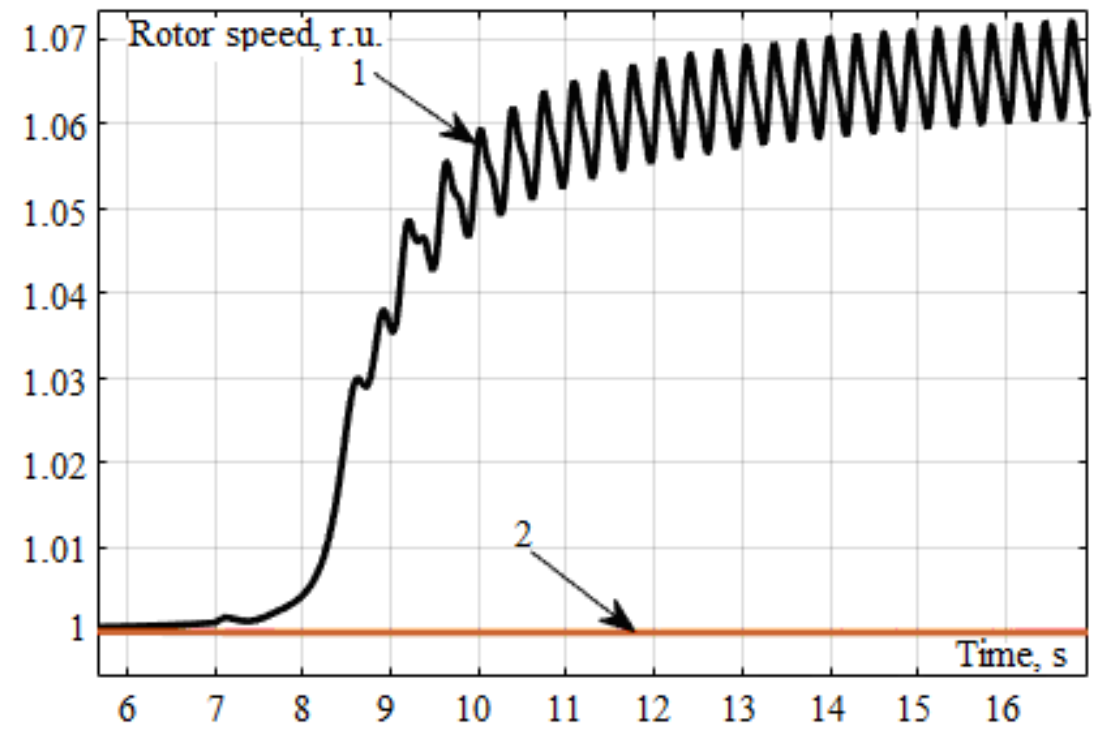

Fig. 11. Oscillograms of the turbogenerator rotor speed: 1 - generators were operated without AVR and ASR; 2 - generators were operated with the use of prognostic AVR and ASR.
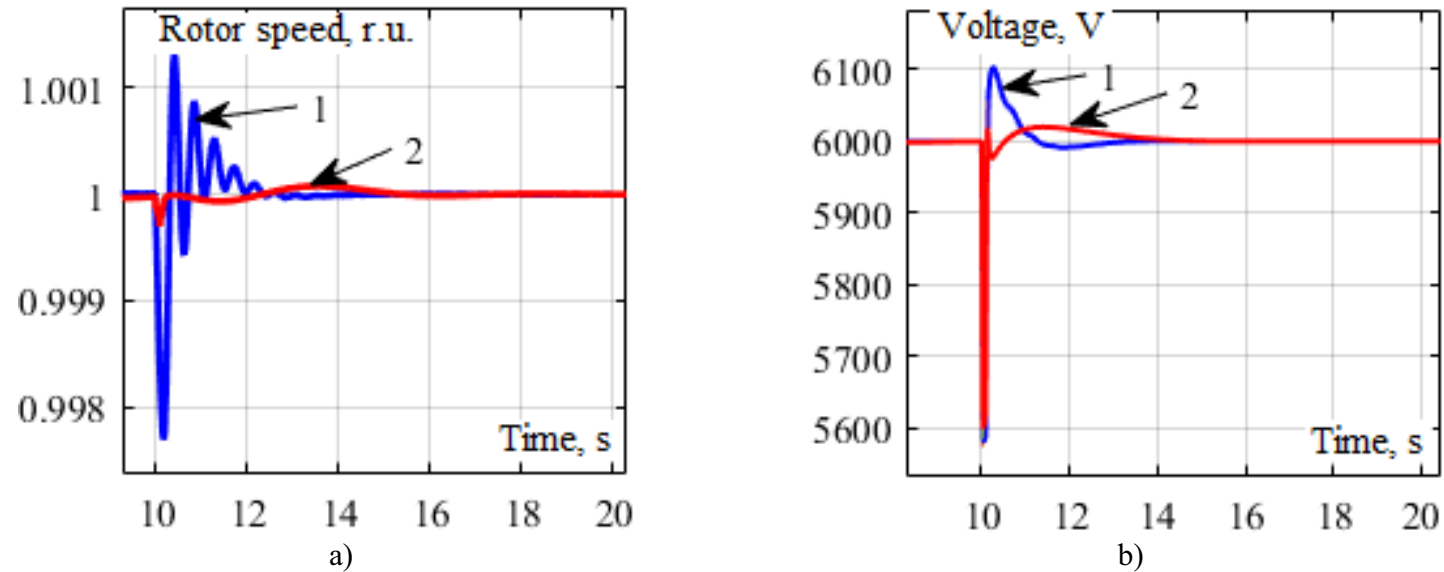

Fig. 12. Oscillograms of the generator rotor speed (a) and the voltage in the DG plant connection assembly (b): 1 - the generators worked with AVR and ASR with concordant adjustment; 2 - the generators were operated using prognostic concordantly adjusted AVR and ASR 

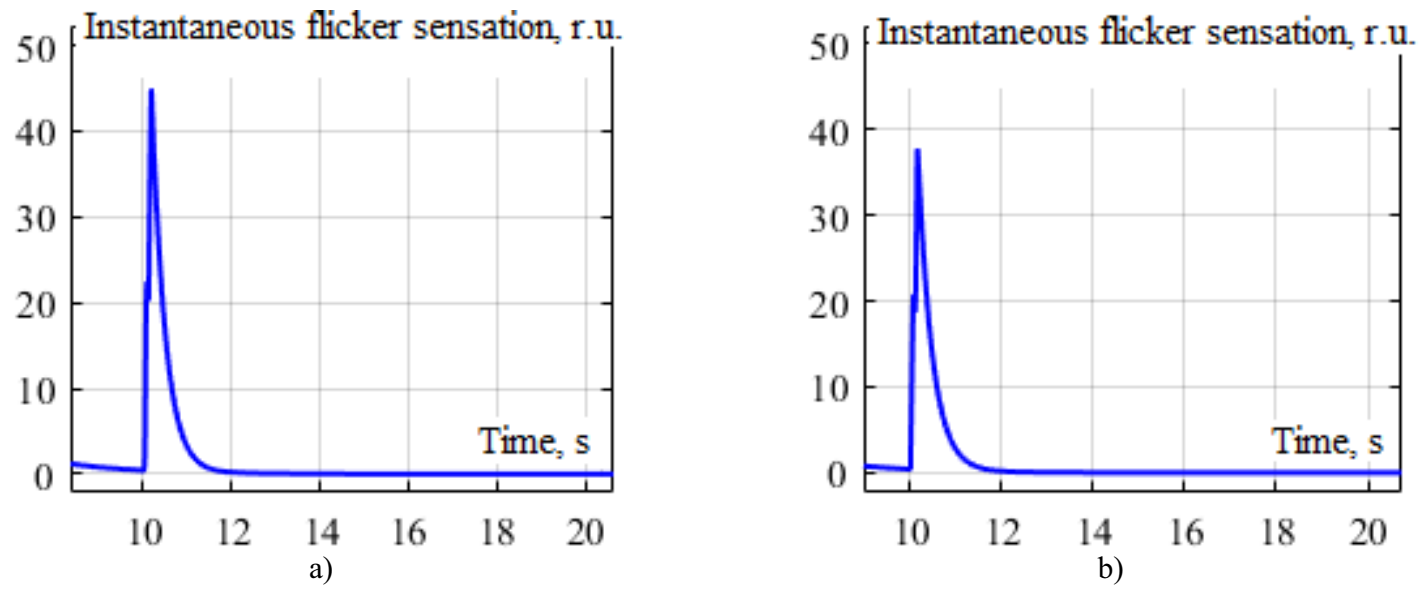

Fig. 13. Flickermeter reading (instantaneous flicker dose): a) the generators were operated with AVR and ASR with concordant adjustment; $b$ ) the generators were operated using prognostic concordantly adjusted AVR and ASR.

\section{Conclusion}

Based on study conducted, the following conclusions can be made:

1. At sharp disturbances caused by switching on and off an additional load, a flicker effect is observed in networks with unregulated DG plants operating based on synchronous generators. Based on the wavelet transformation and spectral analysis methods, it was found that the power spectral density of the generated flicker-noise is inversely proportional to the frequency.

2. The use of look-ahead control algorithms to control the rotor speed and excitation of the DG plants turbine generators makes it possible to increase stability and completely eliminates the occurrence of flicker even without using the regulators adjustment procedure.

3 . The combined use of the procedure for concordant adjustment of regulators of synchronous generators and look-ahead control algorithms makes it possible to solve the issue of flicker occurrence in case of sharp disturbances in the DG plants connection assemblies. In this case, the quality indicators of PSS transient processes with DG plants are significantly improved.

\section{References}

1. Yu.I. Morzhin, Yu.G. Shakaryan, Yu.N. Kucherov, Smart Grid Concept for Unified National Electrical Network of Russia, CD. Preprints of proceedings of IEEE PES Innovative Smart Grid Technologies Europe, Manchester Dec. 5-7 2011. Manchester, GB: IEEE, The University of Manchester, Panel session 5D, (2011).

2. F.N. Mohsen, M.S. Amin, H. Hashim, Application of smart power grid in developing countries, IEEE 7th International Power Engineering and Optimization Conference (PEOCO), (2013)

3. B.M. Buchholz, Z.A. Styczynski, Smart Grids Fundamentals and Technologies in Electricity Networks, Springer-Verlag Berlin Heidelberg, (2014)
4. N.I. Voropay Distributed generation in electric power systems,

http://www.combienergy.ru/stat/983-

Raspredelennaya-generaciya- $\mathrm{v}$ -

elektroenergeticheskih-sistemah (date of treatment 01/09/2020)

5. Yu.N. Bulatov, A.V. Kryukov, Optimization of automatic regulator settings of the distributed generation plants on the basis of genetic algorithm, 2nd International Conference on Industrial Engineering, Applications and Manufacturing (ICIEAM). IEEE Conference Publications, (2016).

6. Yu. Bulatov, A. Kryukov, Van Huan Nguyen, Duy Hung Tran, Fuzzy Controller of Rotation Angle of Blades of Horizontal-Axial Wind Power Generation Plant, Advances in Intelligent Systems and Computing, 983, 105 (2019)

7. Yu.N. Bulatov, A.V. Kryukov, N. Van Huan, Effect of distributed generation plants' automatic controllers on power quality factors, E3S Web of Conferences 114, (2019)

8. N. Jenkins, R. Allan, P. Grossley, D. Kirschen, G. Strbac, Embedded Generation, London; IEEE, (2000)

9. Ph. P. Barker, R.W. De Mello, Determining the Impact of Distributed Generation on Power Systems: Part 1 - Radial Distribution Systems, IEEE PES Summer Meeting, Seattle, WA, USA, July 11$15,222(2000)$

10. Y.N. Bulatov, A.V. Kryukov, K.V. Suslov, Solving the flicker noise origin problem by optimally controlled units of distributed generation, Proceedings of International Conference on Harmonics and Quality of Power (ICHQP) (2018)

11. P.M. Anderson, A.A. Fouad, Power System Control and Stability. Second Edition. IEEE Press, (2003)

12. Yu.N. Bulatov, A.V. Kryukov, N. Van Huan, Forecasting controls for the distributed generation plants, Systems. Methods. Technology, 1 (29), 63 (2016)

13. Yu.N. Bulatov, A.V. Kryukov, N. Van Huan, Automatic prognostic regulators of distributed 
generators, International Multi-Conference on Industrial Engineering and Modern Technologies (FarEastCon), (2018)

14. Digital spectral analysis: parametric, nonparametric, and advanced methods, edited by Francis Castanié, ISTE Ltd, John Wiley \& Sons, Inc. (2011)

15. R.F. Voss, 1/f (Flicker) Noise: A Brief Review, 33rd Annual Symposium on Frequency Control, IEEE, 40 (1979)

16. I.I. Gorban, Statistically unstable processes: connection with flicker, nonequilibrium, fractal and color noise, Proceedings of universities. Radio electronics. 3(55), 3 (2012) 